\title{
A Resource Reservation Protocol with Linear Traffic Prediction for OBS Networks
}

\author{
Ioannis Karamitsos and Chris Bowerman \\ Department of Computing, School of Engineering, University of Sunderland, Sunderland, SR1 3SD, UK \\ Correspondence should be addressed to Ioannis Karamitsos; ioannis.karamitsos@research.sunderland.ac.uk
}

Received 7 April 2013; Revised 19 June 2013; Accepted 20 June 2013

Academic Editor: Zoran Ikonic

Copyright ( 2013 I. Karamitsos and C. Bowerman. This is an open access article distributed under the Creative Commons Attribution License, which permits unrestricted use, distribution, and reproduction in any medium, provided the original work is properly cited.

\begin{abstract}
This paper addresses the issue of providing resource reservation mechanism for OBS networks. We propose a linear prediction mechanism based on least mean square (LMS) method to reduce the burst delay at edge nodes. A reservation method is proposed to increase the reservation probability and to improve the delay reduction performance.
\end{abstract}

\section{Introduction}

With recent technologies, advanced optical networks are used to support the continuously growing IP traffic as they offer huge capacity. Currently, the optical switching and the optical transport are the most interest topics for study and research. After the legacy of SONET/SDH networks, ITU-T Study Group 15 has published a recommendation G.709 for an optical transport network (OTN). The main idea behind an OTN was to build the transport layer around the old SONET/ SDH network, but with less overhead. However, all OTN equipment functionality is based on time slot management and remains incapable of packet or burst recognition. In addition, OTN equipment allows switching at optical layer using reconfigurable optical add/drop multiplexers (ROADMs). The main function of ROADMs addresses the adding or dropping of wavelength channels in WDM systems using either automated lambda processing or simple optical filter devices offering optical-to-electrical-to-optical (O/E/O) conversion and add/drop capability. However, the need for high performance switching of finer wavelength granularities is driving optical packet switching (OPS) and optical burst switching (OBS), two of the optical processing techniques studied in the last years.

OPS provides the finest switching granularity; OBS combines the best characteristic of coarse-grained optical wavelength switching and fine-grained optical packet switching, while avoiding their deficiencies.
OBS transfers transparently the data burst while the control packet is processed in the electronic domain. OBS will need no buffering of the data at intermediate nodes as opposed to optical packet switching (OPS) and achieves efficient bandwidth use appropriate for bursty traffic as in OPS. OBS can be considered as an alternative optical transport technology for the next generation of optical Internet [1]. In IP over OBS networks, the Internet traffic needs to support different types of services to provide QoS and traffic engineering.

Optical burst switching (OBS) [2] allows switching of data channels in the optical domain, where all the electronic processing is performed at the ingress/egress nodes. The main characteristic of the OBS is that it setup dynamically a wavelength path across the network. A separate control packet precedes each burst, which is offset in time with respect to the data packet, carrying forwarding information. The OBS network does not need a two-way reservation and end-to-end signaling like circuit switching, and therefore, the data is transferred through the network without waiting for the setup of the entire connection. IP over OBS WDM networks uses two types of nodes: edge nodes and core nodes abilities and to assign the network resources to optimize the overall routing and performance of the networks as illustrated in Figure 1. An ingress edge node collects IP packets from different sources and assemblies into bursts and also generates the control packet (CP), which is responsible for reserving the resource along the optical path. The main 


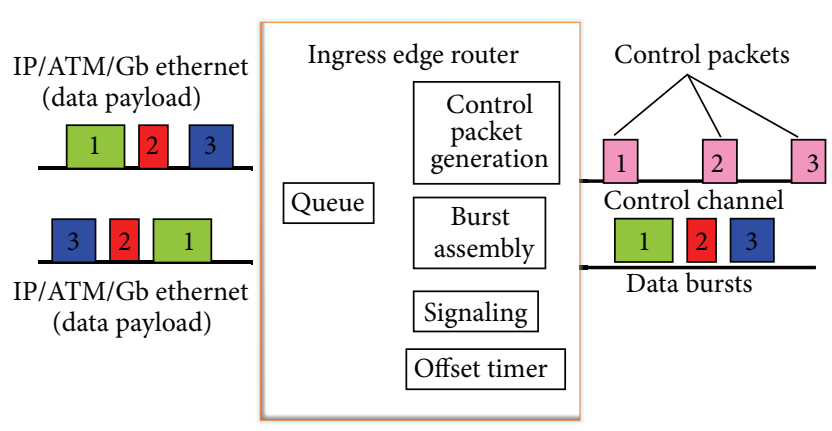

FIGURE 1: OBS edge node.

components of an ingress edge node are burst assembly, signaling, generating the CPs, and determination of the offset time.

The assembly mechanism is responsible for the grouping of several IP packets in a single data burst and then the burst is transferring in the optical network. This approach is more efficient than to handle separately the IP packets in optical network timescale. During the assembly technique of packets, many IP packets are grouped in the ingress of the network. End-to-end delay of IP packets is constituted by four factors: the delay in assembling nodes entering the network, the installation of the path delay due to control packets, the transmission time of IP packets, and the propagation delay in the core network. The last two factors delay (transmission time and propagation delay) depends on the selected path and the available bandwidth in the network backbone. Mainly, the contribution of this paper aims to explain and analyze the techniques, which are used for reduction of the average delay of the packets during assembly in bursts. These techniques actually use a linear prediction filter to estimate the number of packets that enter the node where assembly takes place during the next period. This time is used by the control packet (CP) for the reservation of necessary resources to the core network before the burst transmission starts.

This paper is organized as follows: in Section 2 a description of least mean square linear predictor is introduced. The JET reservation without prediction in Section 3 is presented. In Section 4, we describe the fast reservation mechanism using linear prediction based on least mean square (LMS) algorithm. A new resource reservation scheme mechanism is presented in Section 5, and simulation results are presented in Section 6.

\section{Linear Prediction Methodology}

In this section, we examine the linear traffic predictor for the optical burst switching networks. The reason for using linear predictors in OBS networks is that a quantitative estimation (accuracy is not necessary) is required, thereby improving overall networks performance. Unlike the optical packet switching, the assessment of the linear predictors taken is required to be more accurate because the efficiency of the packet switching is limited by the distribution of arrival of packets (ingress) within the prediction time period.
While the length of IP packets is dynamically variable and the Internet traffic presents a long-range dependence, nonstationarity, and multifractal scaling in short timescales, a traffic prediction method is required. Recently, significant changes have occurred in terms of understanding the nature of the network traffic. It has been accepted by various studies [3-5] that many types of traffic have the status of long-range dependence (LRD). The history of a long-range dependence process has a significant influence of this value. Moreover, the correlation matrix that accompanies such traffic implies that the present intense traffic burstiness could be characterized by the term of self-similarity. Based on the previous characteristics, it can be argued that the application of linear algorithmic predictions based on stochastic traffic structures, which consist of long-range dependent process, would not bring the desired performance result. However, in [5] the use of linear prediction algorithm concerning Internet traffic without the necessity to examine in depth the history of the traffic is described. This is because, as explained in [5], shortterm correlations are those which dominate the efficiency of model predictive control (MPC) and the effect of long-range dependence is rather minimum in realistic networks.

A solution to this problem would be the possibility of predicting the future behavior of the traffic, that is, the possibility of assessing the number of packets entering the queue (ingress) the next time " $t$ ". In this way the assembly algorithm will "know" whether to wait or not for subsequent packets and adapt the waiting period of the burst in the queue.

The target of traffic prediction is to predict as accurate as possible the future volatility of the frequency of traffic rate, which can be applied in various scenarios of Internet or network design. The applicability of traffic prediction analysed in [6] examines how in the future a prediction can be made and what is the minimum error with a specific prediction time interval. The quality of the prediction is determined with the imprecision that characterises the forecast and measure of the dispersion of forecasted errors. The inaccuracy depends on various factors such as the size of the traffic history, the kind of the prediction, and the type of traffic to which it applies. In [6], it was shown that the performance of traffic prediction and its implementation are limited by the accuracy of the estimates, which worsens as the prediction intervals are getting bigger.

For real time applications, the prediction process shall be carried fairly quickly and with the minimum percentage of stored traffic information. Also, the time of a prediction should be selected to include not only the time required to process control information but also the round-trip delay. Finally, the process of forecasting should be as precise as possible so as not to reserve and consume all the resources of the network. Nevertheless, the process has to maintain the overall quality provided by the traffic management function.

\section{Least Mean Square Linear Predictor}

Suppose that $S(k)$ is the size of $k$ th burst in bytes and $t(k)$ is the time between the creation of assembling the packets that constitute it as shown in Figure 2. 


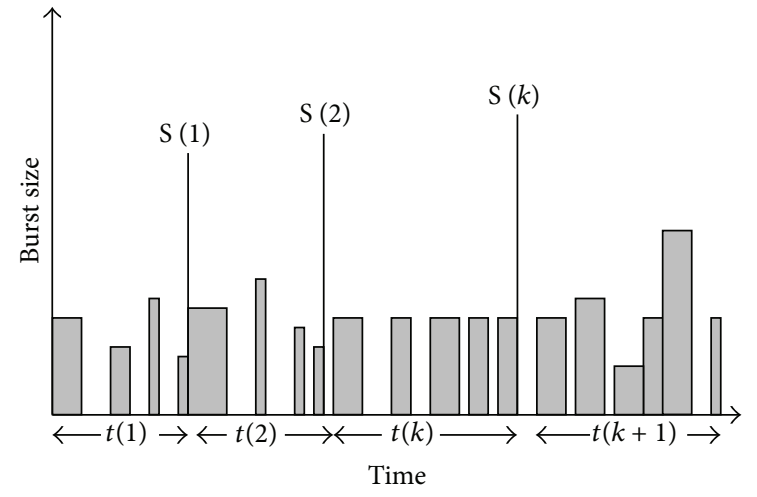

Figure 2: Values of size and time duration during burst assembly process.

If these two values were known from the beginning of the assembly, the allocation of resources would have been possible so as to reduce the transmission delay due to burstification. To simplify the calculations, it is assumed that the $t(k)$ is known and what needs to be determined is the burst length $S(k)$.

In the following, for the burst prediction a linear algorithm is used [3], which is the least mean square (LMS). Actually, LMS is one of the simplest linear adaptive algorithms, which shows good performance at small computational cost as illustrated in Figure 3.

Let $S_{d}(k)$ is the length of the $k$ th burst, then the length of the next incoming burst is predicted according to the previous $N$ bursts and is equal to

$$
\widetilde{S}_{d}(k+1)=\sum_{i=1}^{N} w_{i} \times S_{d}(k-i+1),
$$

where $w_{i}, i=1, \ldots, N$, are the coefficients of the filter and are updated by the LMS algorithm. A control header of the control packet makes an advance reservation according to the predicted value of $\widetilde{S}_{d}(k+1)$. The advance reservation length is denoted as $S_{R}(k+1)$, and if it is optimal it should be equal to the actual burst length.

Suppose that the reservation length is equal to the predicted length and a smaller prediction of burst length is defined as

$$
e(k+1)=S_{d}(k+1)-\widetilde{S}_{d}(k+1)
$$

and will result as insufficient reservation of optical path. This requires to $\mathrm{CP}$ to be retransmitted after the burst assembly process finishes.

The reservation length has been defined as

$$
S_{d}(k+1)=\widetilde{S}_{d}(k+1)+\delta,
$$

where $\delta$ is the small margin of correction. The value of $(\delta)$ has effect on both probabilities of successful engagement by the CP (the bigger the value of $\delta$, the smaller the period during which the capacity committed is not used).

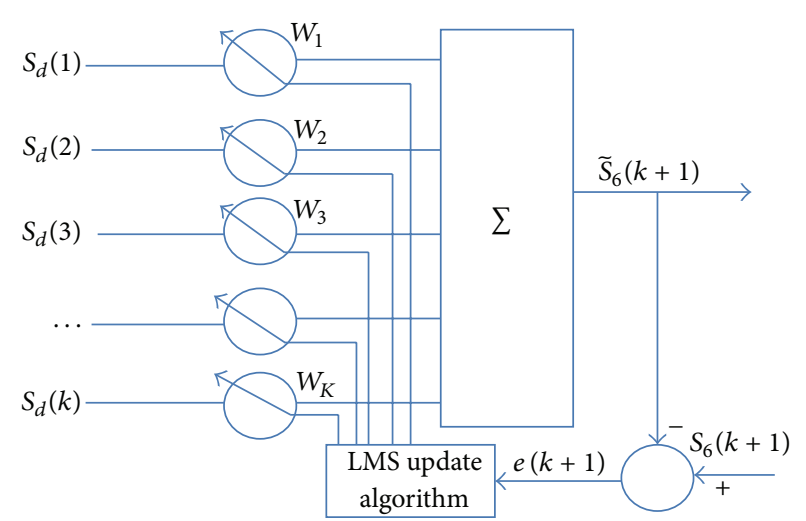

FIgURE 3: LMS Algorithm.

To get the best possible chance of success without significantly increasing system costs, $\delta$ is chosen to be a multiple of the root mean square (RMS) remainder of $(e)$ of the filter:

$$
\delta=c_{\delta} \sqrt{\frac{\sum_{i=1}^{N} e^{2}(k-i+1)}{N}},
$$

where $c_{\delta}$ is the constant that takes small values (e.g., less than 2 or 3 ) and is considered as a correction parameter, a trade-off between the bandwidth and the successful CP pretransmission probability. The LMS pseudocode is presented as follows.

\section{LMS Pseudocode Algorithm}

$$
\begin{aligned}
& \text { //Inputs parameters: } \\
& h \text { : filter order } \\
& \mu \text { : step size } \\
& S_{d} \text { : input vector } \\
& \text { //Initialization: } \\
& w(0)=0 ; \\
& \text { for } k=0,1,2, \ldots \\
& \widetilde{S}_{d}(k+1)=w^{T}(k) * S_{d}(k) \\
& e(k+1)=S_{d}(k+1)-\widetilde{S}_{d}(k+1) \\
& w(k+1)=w(k)+\mu e(k) S_{d}(k)
\end{aligned}
$$

end.

\section{JET Reservation Protocol}

JET was proposed by Yoo et al. [7] in 1997, and it is the most prevailing distributed protocol for OBS networks. JET is also a one-way reservation protocol. Unlike JIT [8], JET [9] adopts delayed reservation; that is, JET uses estimated configuration with the information embedded in the control packet.

JET reservation protocol [9] works as follows: the reservation starts at the expected arrival time of the burst, instead of the control packet's arrival time. A setup release message is released by the source when the burst assembly has been finished. The process of JET is illustrated in Figure 4. 


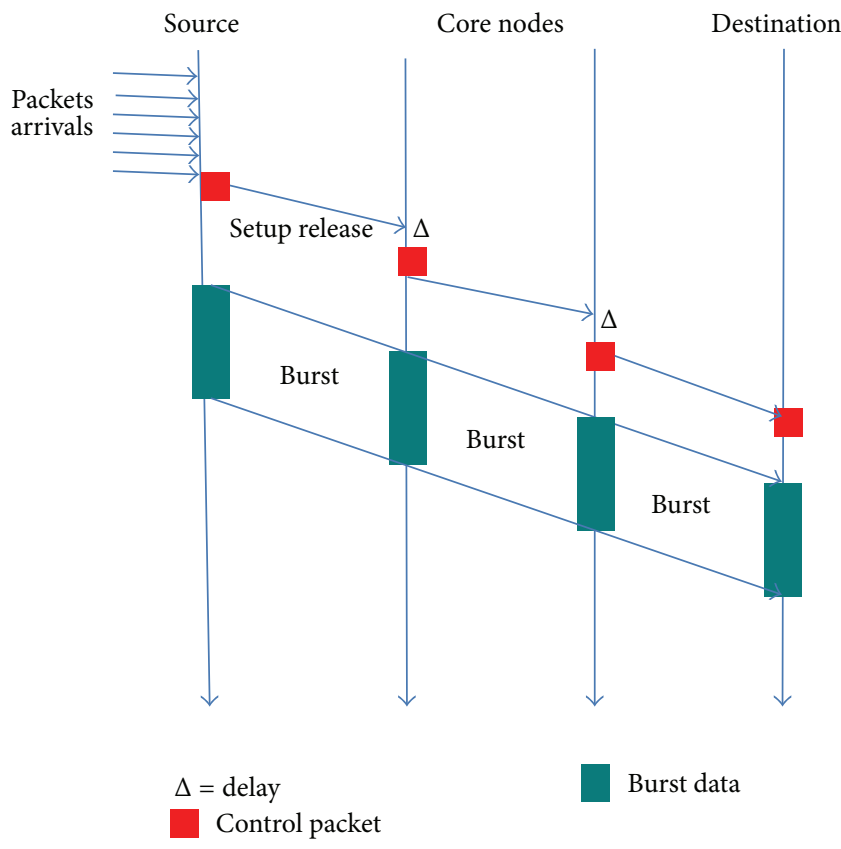

FIGURE 4: JET reservation process.

The control packet is sent to the destination after the burst assembly. The control packet is processed at each intermediate node to reserve the resource. The switch is configured for the burst according to the information embedded in the control packet, such as the offset time. The switch releases the resource according to the estimated burst departure time. JET can achieve better blocking performance from the other mechanisms.

JET is based on the delayed reservation process and allows the implementation of a non-first come first served (nonFCFS) service. Figure 5 shows how two consecutive bursts are scheduled over the same data channel following the JET protocol.

The setup message for burst A arrives first followed by the message setup for burst B. As seen from Figure 5, the offset time of burst $A$ is much longer than the offset time of burst B. Upon reception of the setup message, the switch notes the later arrival of burst $\mathrm{A}$, but does not start connections in the switching circuit. After burst A has been received, a blank time period is noted which is the time until the switch starts to configure the switching circuit for the burst A time $\left(t_{6}-T_{\text {OXC }}\right)$.

Suppose that in $t_{2}$ arrives the setup message for the burst $B$ and no other burst transmission has been timely set up. With the arrival of the message setup for burst B time $t_{2}$, the switch notes that the burst $B$ arrives before the arrival of burst A and executes an algorithm of gaps filling [10, 11] to determine whether the new burst can be accepted or not. In order to accept the burst, there should be enough time between the end of transmission of the burst $B$ and the arrival of the burst A. For this scenario, as shown in the Figure 5, the burst $B$ is accepted and the service is completed before the start of switching circuits for burst A. However, due to the fact

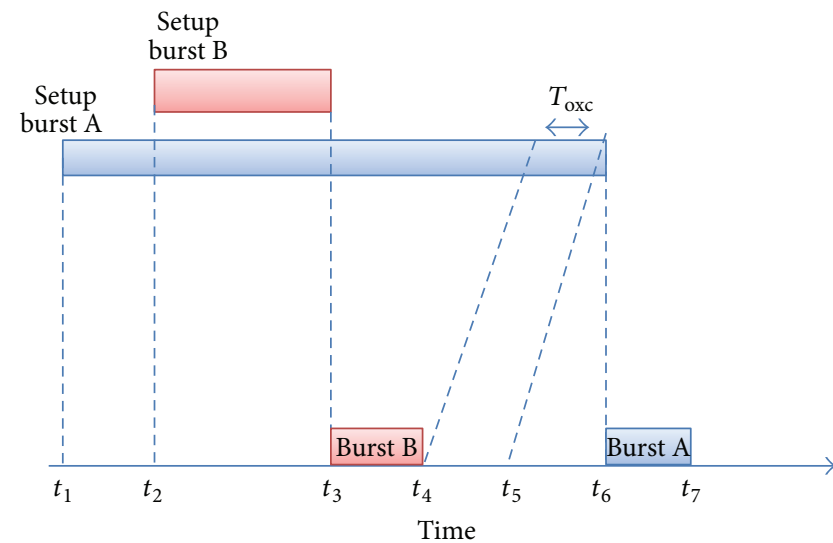

FIGURE 5: Non-FCFS scheduling flow using JET protocol.

that the setup message for burst $B$ arrived after the respective one for burst $\mathrm{A}$, this operation resulted in a non-FCFS service of bursts.

\section{A New Resource Reservation Mechanism Using LMS Linear Predictor}

In this section, a resource reservation mechanism is presented using the LMS algorithm [3] for the prediction of the bursts. In this mechanism, the setup release message is transmitted before the burst has been assembled. The new resource reservation mechanism works as follows.

(i) Initialization Phase. The control unit (CU) predicts the reservation length for the incoming data burst. The prediction is derived using the LMS algorithm.

(ii) Set-Up Phase. When the first packet arrives to burst assembly queue, the $\mathrm{CU}$ unit provides the necessary information regarding the path and the predicted reservation length in the control packet $(\mathrm{CP})$ header. Then the $\mathrm{CP}$ is pretransmitted in the OBS core node.

(iii) Process Phase. When the burst assembly process is finished, the actual burst length is compared with the predicted reservation length and one of the two following cases may occur:

(a) if the actual burst length is less than or equal to the predicted length, then the CP pre-transmission is considered a success;

(b) if the actual burst length is greater than the predicted length, the $\mathrm{CP}$ pre-transmission is a failure. The $\mathrm{CP}$ has to be retransmitted for this burst at a later time with the actual burst length and the data payload followed by an additional offset time. 


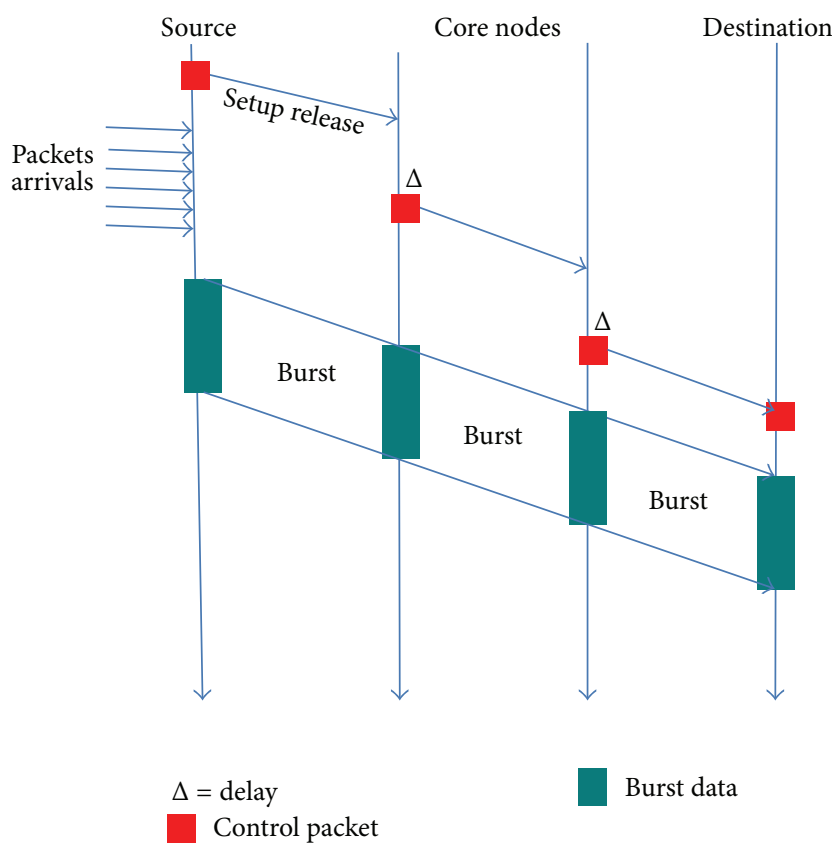

FIGURE 6: Resource reservation process using linear prediction.

The process of the new resource reservation mechanism is illustrated in Figure 6.

The proposed mechanism is presented with the following pseudocode algorithm.

\section{Algorithm Pseudocode}

//Initialization phase;

LMS filter predicts reservation burst length $\widetilde{S}_{d}(k+1)$ for the $(k+1)$ next burst.

//Set-up phase;

The packet inserts to burst assembly queue $Q$;

If the assembly queues is empty

$$
\begin{aligned}
& \text { then } L=1 ; \\
& \text { else } L=L+1 ;
\end{aligned}
$$

end.

//Process phase;

if $\widetilde{S}_{d}(k+1)<S_{d}(k)$

then burst assembly complete; send control packet to control channel; send burst data after an offset;

end.

\section{Simulation}

We performed our simulation using NS-2 platform program for a simple OBS network and more complex ultra long-haul (ULH) network.

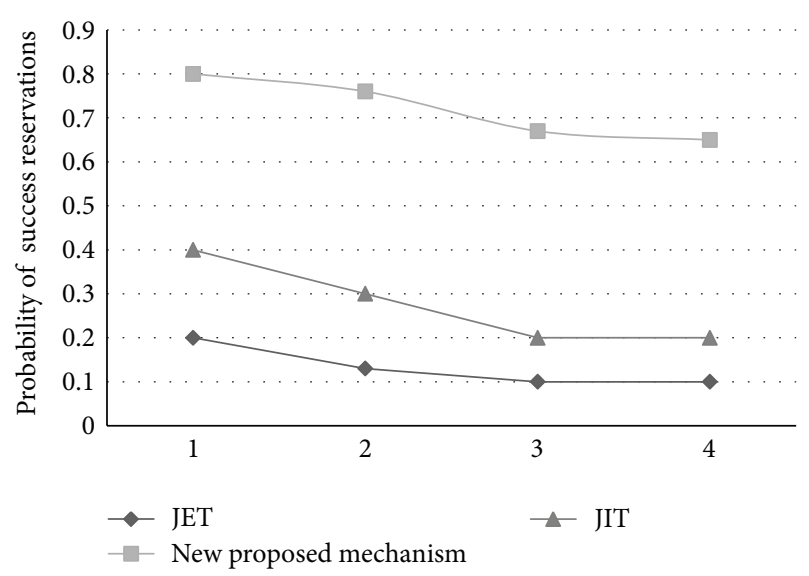

FIGURE 7: Comparison of success reservation process versus shape parameter $(a)$.

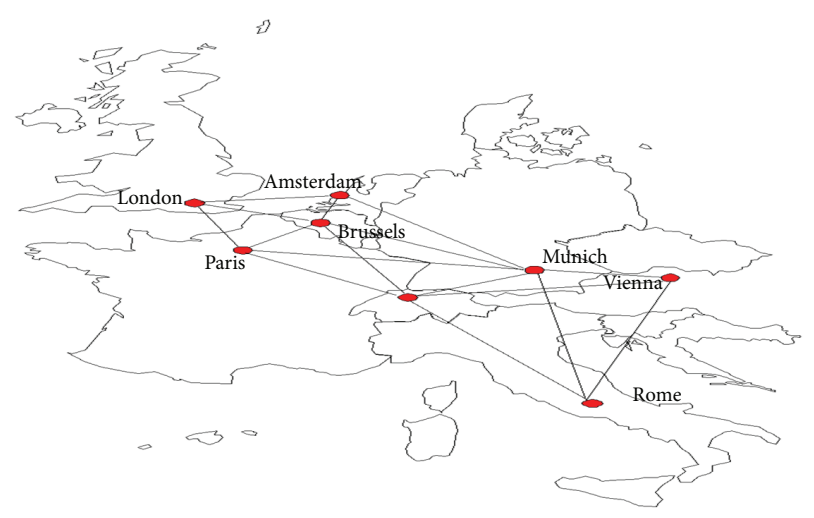

FIGURE 8: Simulation complex network.

The OBS network consists of three edges and two core nodes with a link bandwidth per channel equal to $40 \mathrm{Gbps}$. In this paper, we use LMS filter to predict the burst length for the proposed algorithm in comparison with the traditional JET and JIT reservation algorithms. In each simulation, we change the shape parameter $(a)$ of the Pareto distribution using the following value $a=1,2,3,4$.

In our proposed algorithm, we use one LMS linear predictive filter in order to predict the length of the next incoming data burst. Figure 7 shows the probability of successful reservation using a burst length correction parameter $c_{\delta}$ equal to 2 .

From Figure 7 we observe that the success probability is quite high in amount of 0.8 and decreases when the shape parameter $(a)$ is increasing. On the other hand, the two JIT and JET algorithms have a low success probability of 0.3 but steady curves in terms of shape parameters.

In this section, we are going to simulate more complex networks such as an ultra long-haul (ULH) network. This network consists of 8 nodes connected with 16 bidirectional links. These 8 nodes correspond to 8 different major and highly European cities, which according to studies that have been conducted are considered to be the main points 


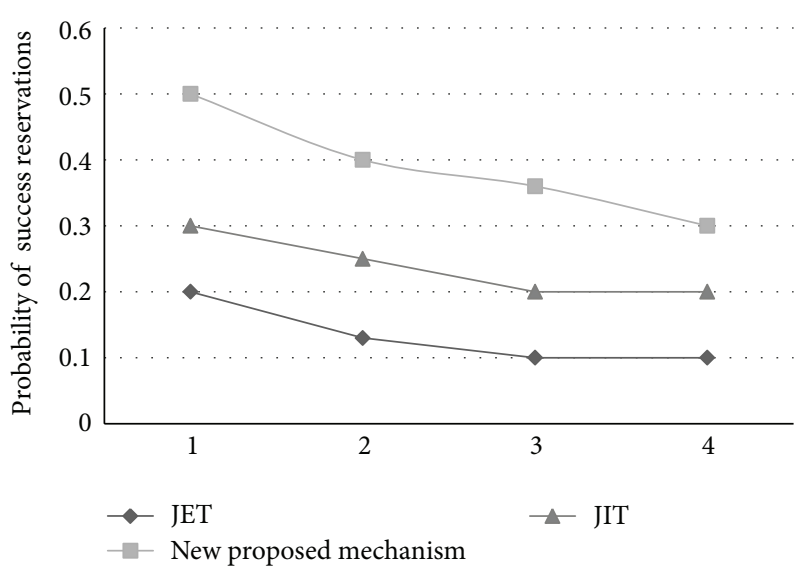

FIGURE 9: Comparison of success reservation process versus shape parameter $(a)$.

of traffic grooming and thus contribute significantly to the traffic circulating in the PAN-network. We use LMS filter to predict the burst length for the proposed ULH network. We assume the following: (a) Poisson packets arrivals, (b) exponential packet holding time, (c) uniform distribution of ingress-egress nodes for each bursts, (d) shortest path routing of wavelengths, and (e) loss of blocked bursts. For each source destination pair of nodes, first the routing takes place by the selection of the path with the least BER $=10^{-12}$ (or best OSNR) and then the wavelength assignment follows. The topology of the network is given by Figure 8 .

Figure 9 shows the probability of successful reservation using a burst length correction parameter $c_{\delta}$ equal to 2 .

From Figure 9 we observe that the success probability for our proposed mechanism is low in amount of 0.5 and decreases when the shape parameter $(a)$ is increasing. The main reason is that the reservation process is very complicated when we have many hops but the success rate remains acceptable than the other two reservation mechanisms such as JIT and JET.

\section{Conclusion}

In this paper, a resource reservation mechanism using LMS linear predictor is presented. The study is based on traditional linear algorithm and the benefit is the delay reduction of bursts. For the future work, we need to provide simulations and comparison between the proposed mechanism and others OBS reservations schemes for more complex networks taking into consideration the physical linear and nonlinear physical impairments.

\section{References}

[1] C. Qiao and M. Yoo, "Optical burst switching (OBS) - a new paradigm for an Optical Internet," Journal of High Speed Networks, vol. 8, no. 1, pp. 69-84, 1999.
[2] Y. Chen, C. Qiao, and X. Yu, "Optical burst switching: a new area in optical networking research," IEEE Network, vol. 18, no. 3, pp. 16-23, 2004.

[3] D. Mortato, J. Aracil, L. A. Diez et al., "On linear prediction of Internet traffic for packet and burst switching networks," in Proceedings of 19th International Conference on Computer Communications and Networks (ICCCN '01), pp. 138-143, October 2001.

[4] J. Liu, N. Ansari, and T. J. Ott, "Frr for Latency Reduction and QoS provisioning in OBS Networks," IEEE Journal on Selected Areas in Communications, vol. 21, no. 7, pp. 1210-1219, 2003.

[5] S. A. M. Ostring and H. Sirinena, "The influence of long-range dependence on traffic prediction," in Proceedings of the IEEE International Conference on Communications (ICC '01), 2001.

[6] A. Sang and S.-Q. Li, "Predictability analysis of network traffic," in Proceedings of the 19th Annual Joint Conference of the IEEE Computer and Communications Societies (INFOCOM '00), pp. 342-351, March 2000.

[7] M. Yoo, M. Jeong, and C. Qiao, "A high speed protocol for bursty traffic in optical networks," in All-Optical Communication Systems: Architecture, Control, and Network Issues III, vol. 3230 of Proceedings of the SPIE, pp. 79-90, November 1997.

[8] Y. Xiong, M. Vandenhoute, and H. C. Cankaya, "Control architecture in optical burst-switched WDM networks," IEEE Journal on Selected Areas in Communications, vol. 18, no. 10, pp. 1838-1859, 2000.

[9] J. Xu, C. Qiao, J. Li, and G. Xu, "Efficient channel scheduling algorithms in optical burst switched networks," in Proceedings of the 22nd Annual Joint Conference on the IEEE Computer and Communications Societies (INFOCOM '03), pp. 2268-2278, April 2003.

[10] K. Ioannis and V. Evangelos, "A survey of Reservation schemes for Optical Burst Switching (OBS)," WSEAS Transactions on Circuits and Systems, vol. 2, no. 2, pp. 395-400, 2003.

[11] R. Faulhaber, "Design of service systems with priority reservation," in Proceedings of the IEEE International Conference on Communications, pp. 3-8, 1995. 

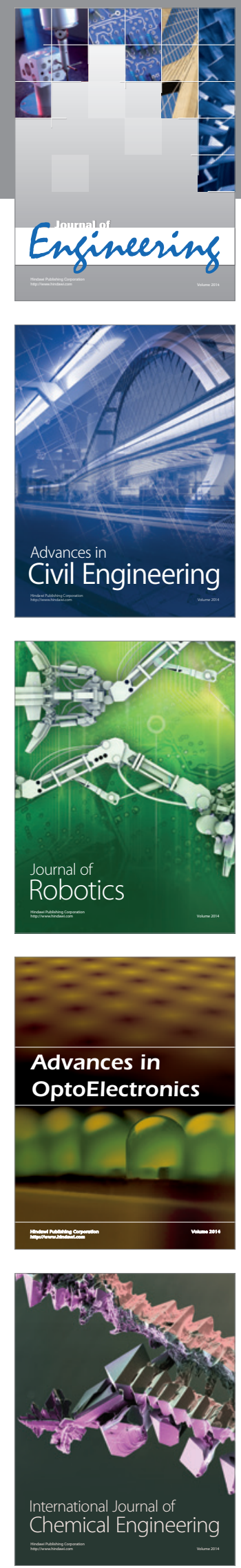

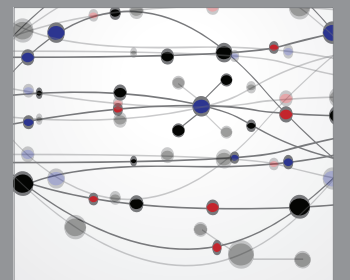

The Scientific World Journal
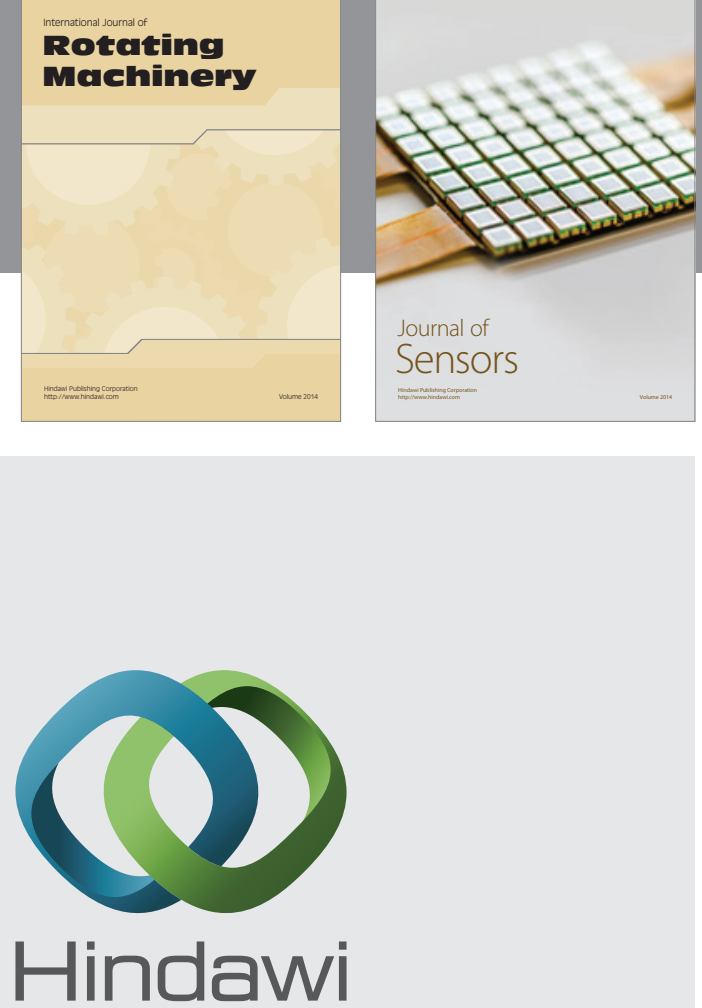

Submit your manuscripts at http://www.hindawi.com
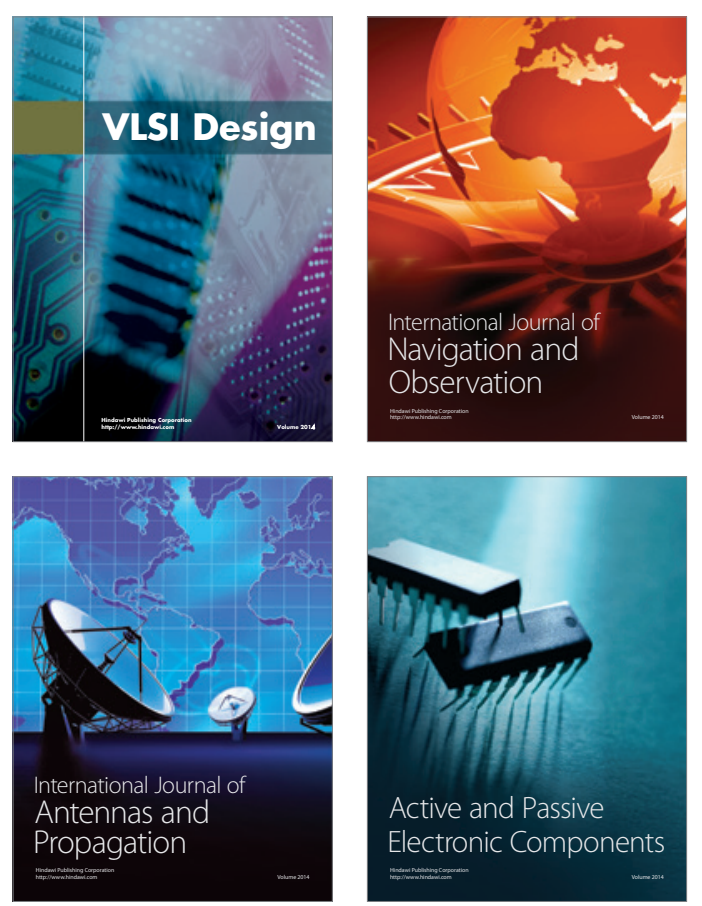
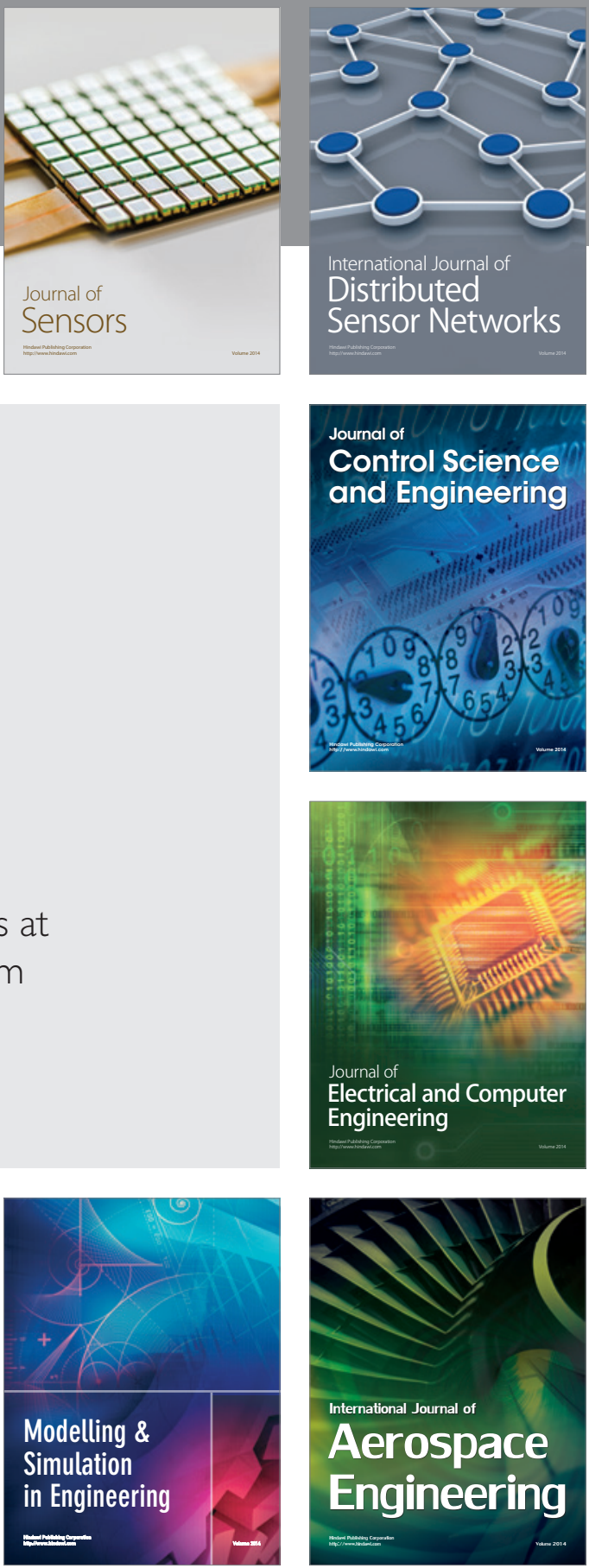

Journal of

Control Science

and Engineering
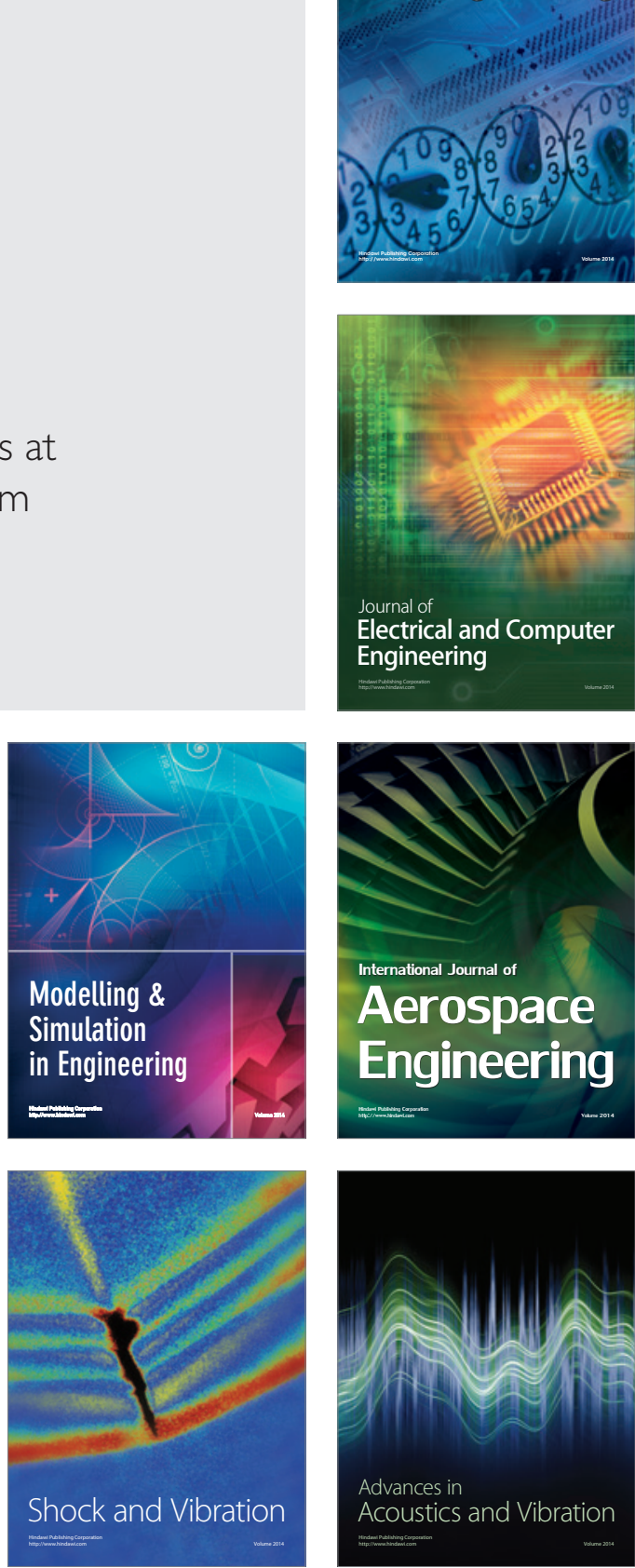